\title{
Iron L $\alpha$ and L $\beta$ X-ray Lines: a Comparison of EPMA Measurements and Theoretical Calculations, With Possible Implications for Oxidation Determination
}

\author{
Aurélien Moy ${ }^{1}$, John Vinson ${ }^{2}$ and John Fournelle ${ }^{1}$ \\ 1. Department of Geoscience, University of Wisconsin, Madison, USA. \\ 2. Material Measurement Laboratory, National Institute of Standards and Technology, Gaithersburg, USA.
}

In the past decade, field emission gun (FEG) electron probe microanalysis (EPMA) has allowed the reduction of the electron beam size leading to sub-micron resolution imaging. However, to improve the lateral spatial resolution of quantitative analysis, not only the electron beam diameter must be reduced but also the interaction volume of the electrons. This is done by decreasing the accelerating voltage from typically $15 \mathrm{kV}$ to $5-7 \mathrm{kV}$, or even lower. However, at low accelerating voltage, the typically used X-ray lines (the $\mathrm{K} \alpha$ lines) of most of the elements of the periodic table cannot be excited. Therefore, to analyze these elements, the L lines must be used. However, for most of these elements, the emission of the L Xray lines is produced by an electronic transition involving an "outer electronic shell" belonging to the valence band. In the case of the transition metals, the $L \alpha$ and $L \beta$ X-ray lines involve a partially filled $3 d$ electron shell bonded with electrons from the neighboring atoms. Consequently, a change in the composition of the material induces a change in the state of the outer electronic orbitals followed by a change in the electron energies and in the electronic transition probabilities. The atomic parameters of these L lines (mass absorption coefficient, fluorescence yield, Coster-Kronig factor, ...) not only depend of the studied element but also of the composition and nature of the specific material, for both of the unknown and of the standard used to generate the k-ratio.

The present work focuses on the study of the L $\alpha$ and L $\beta$ X-ray lines of the transition metal iron as Fe is found in many materials of interest, and its oxidation state is of importance in many geological materials of interest. A method of determination consists in the study of the energy shift of the L $\alpha$ and L $\beta$ X-ray lines which varies as a function of the degree of Fe oxidation. However, the observation of this shift is difficult because of the strong self-absorption of the main L lines. Indeed, the Fe absorption edges of the $\mathrm{L}_{2}$ and $\mathrm{L}_{3}$ electron sub-shells fall on the high energy side of the $\mathrm{L} \beta$ and $\mathrm{L} \alpha \mathrm{X}$-ray lines, respectively. The recorded spectrum is then distorted and the apparent maximum of the peaks is shifted to the low energies (Fig. 1). To overcome this problem, the accurate knowledge of the mass attenuation spectrum can be used to reconstruct the peak shape as it was before absorption, allowing the determination of the characteristic $\mathrm{X}$-ray energy. However, the mass attenuation spectrum is dependent on the outer electron shells and then changes from one material to another due to the bonding effects. The experimental observation of the Xray emission spectrum before self-absorption can be achieved by EPMA using nm thick films for which the absorption of the X-rays inside the layer is negligible. Two sets of thin films have been made by evaporation under vacuum of pure $\mathrm{Fe}(99.995 \%)$ on an auto-supported $\mathrm{C}$ film previously deposited on copper grids. The main advantage of using an auto-supported thin film as a substrate compared to a bulk substrate is that almost no backscattered electrons are produced in the substrate and then the X-ray intensity emitted by the Fe film is proportional to the thickness. The Fe thicknesses were characterized by measuring the Fe and $\mathrm{C} \mathrm{K \alpha}$ X-ray lines at 10,15,20,25 and $30 \mathrm{kV}$ on the films and on pure bulk standards. The obtained k-ratios were analyzed using the Monte Carlo code PENEPMA [1] in which the $\mathrm{k}$-ratios were simulated for a given thickness of Fe and C. As the thin film X-ray intensity is proportional to the thickness, and so is the k-ratio, only few simulations with different film thicknesses are needed to converge to the thickness reproducing the experimental k-ratios. The thicknesses (for each layer) obtained for the thicker set of samples are Fe: $2.98 \mathrm{~nm}$ and C: $15.8 \mathrm{~nm}$ and for the thinner set of samples, Fe: 0.64 $\mathrm{nm}$ and $\mathrm{C}: 11.5 \mathrm{~nm}$. 
The Fe L $\alpha$ and L $\beta$ spectrum was acquired with a CAMECA SXFive FE probe on the samples using two spectrometers with TAP crystals, at $15 \mathrm{kV}$ and $650 \mathrm{nA}$. Ten spectra were acquired and averaged to reduce statistical fluctuations. Ten spectra were also acquired on a bulk Fe standard. The two averaged spectra are compared on Figure 1. On the low energy side of the Fe L $\alpha$ line, the bulk Fe spectrum follows the shape of the thin film Fe spectrum indicating almost no variation of absorption in this energy range. The spectrum measured on the Fe film can be fitted very well with three pseudo-Voigt functions representing the $L \alpha_{1}, L \alpha_{2}$ and $L \beta_{1}$ X-ray transitions. The strongly absorbed spectrum measured on the bulk Fe sample can then be reconstructed using the functions and by ensuring the reconstructed spectrum fits the experimental spectrum on the low energy side of the Fe L $\alpha$ line. Assuming the same behavior for Fe compounds, the spectrum can be reconstructed and used to find the characteristic energy of the X-ray lines. The energy peak shift, compared to the pure Fe X-ray lines, can then be determined.

The energy shift of the Fe L $\alpha$ X-ray line could be related to the Fe oxidation state by means of experimental calibration curves, obtained by measuring the peak shifts in well characterized standards, or by comparison with theoretical shifts calculated using physical theory like the Density Functional Theory (DFT). This last approach becomes promising with the improvement of the accuracy of the physical models used and with the increase of the available computational power. We used the code OCEAN [2], an ab initio DFT and Bethe-Salpeter Equation (BSE) solver, for the calculation of the core-level spectra of the $\mathrm{L} \alpha$ and $\mathrm{L} \beta \mathrm{X}$-ray lines of pure $\mathrm{Fe}$ and hematite $\left(\mathrm{Fe}_{2} \mathrm{O}_{3}\right)$. The calculated pure Fe spectrum is directly comparable to the spectrum acquired on the Fe thin films. As shown on Figure 2, the energy splitting between the $\mathrm{L}_{2}$ and $\mathrm{L}_{3}$ sub-shells, producing the $\mathrm{L} \beta$ and $\mathrm{L} \alpha$ lines, respectively, is very well reproduced by the calculations. The calculated spectrum tends to overestimate the intensity of the L $\beta$ line compared to the $\mathrm{L} \alpha$ line. This can be explained by the fact that the $\mathrm{L}_{2}$ and $\mathrm{L}_{3}$ sub-shells have different electron ionization cross sections, i.e. different probabilities an ionization occurs in the $\mathrm{L}_{2}$ or $\mathrm{L}_{3}$ sub-shells. The DFT calculation assumes an initial vacancy in these two sub-shells and does not consider the electron ionization process. Creating thin film of Fe compounds, like $\mathrm{Fe}_{2} \mathrm{O}_{3}$, is more difficult than a simple $\mathrm{Fe}$ evaporation. A future investigative method will consist of using an ion beam instrument to ablate a $\mathrm{Fe}_{2} \mathrm{O}_{3}$ sample with a shallow slope to obtain a thin thickness at the edge of the sample and to acquire a spectrum for comparison with the OCEAN calculations. The ultimate aim would be to be able to correlate experimental $\mathrm{Fe} \mathrm{L} \alpha / \mathrm{L} \beta$ spectra to the valence state of Fe using DFT calculations.

\section{References:}

[1] X Llovet and F Salvat, IOP Conf. Series: Materials Science and Engineering 109(1) (2016), 012009.

[2] J Vinson et al, Phys. Rev. B 83 (2011), 115106.

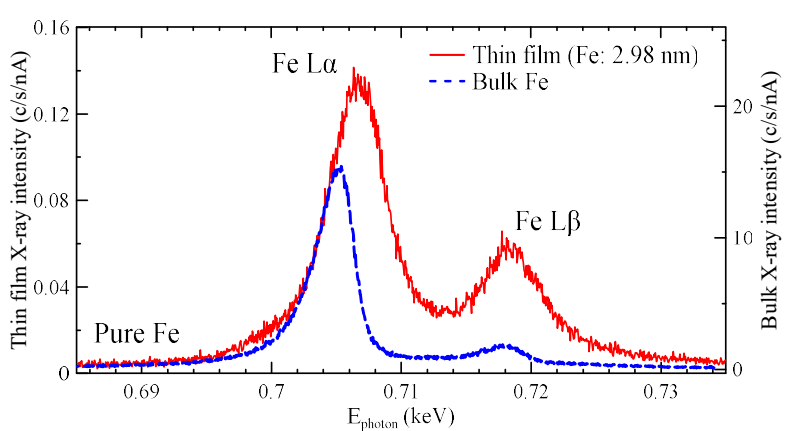

Figure 1. Fe $\mathrm{L} \alpha$ and $\mathrm{L} \beta \mathrm{X}$-ray lines measured on Fe thin film (red) and on pure bulk Fe (blue).

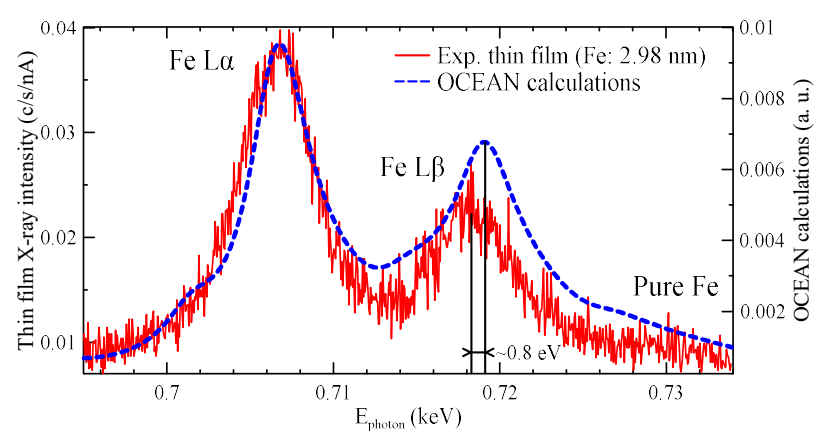

Figure 2. Experimental (red) and calculated (blue) $\mathrm{Fe}$ $\mathrm{L} \alpha$ and $\mathrm{Lb} \mathrm{X}$-ray lines for pure Fe. 